\section{Blind Channel Identification Based on Second-Order Statistics: A Frequency-Domain Approach}

Lang Tong, Member, IEEE, Guanghan Xu, Member, IEEE, B. Hassibi, Student Member, IEEE, and T. Kailath, Fellow, IEEE

\begin{abstract}
In this communication, necessary and sufficient conditions are presented for the unique blind identification of possibly nonminimum phase channels driven by cyclostationary processes. Using a frequencydomain formulation, it is first shown that a channel can be identified by the second-order statistics of the observation if and only if the channel transfer function does not have special uniformly spaced zeros. This condition leads to several necessary and sufficient conditions on the observation spectra and the channel impulse response. Based on the frequency-domain formulation, a new identification algorithm is proposed.
\end{abstract}

Index Terms-Communication systems, system identification, cyclostationary processes.

\section{INTRODUCTION}

Reliable communication often requires the identification of the channel impulse response. Such identification can facilitate channel equalization as well as maximum likelihood sequence detection. The so-called blind channel identification means that the channel is identified without using a training signal; instead, the identification is achieved by using only the channel output along with certain a priori statistical information on the input. Such methods have the potential to increase the transmission capability due to the elimination of training signals. Recently, since the publication of [4], [11], [12], blind channel identification techniques using secondorder cyclostationary statistics have attracted considerable research attention. The purposes of this communication are: i) to investigate basic questions of channel identifiability in the frequency domain and their relations to the time-domain results obtained earlier in [12]; ii) to present a new frequency-domain approach to blind channel identification. A set of necessary and sufficient conditions for channel identifiability is presented. Based on a new optimization criterion, a new channel identification algorithm is derived. Such an algorithm is not easily seen in the time domain and offers potential savings in computation.

Manuscript received May 2, 1993; revised May 17, 1994. This paper was presented at the International Symposium on Information Theory, San Antonio, TX, 1993. This research was supported in part by the Army Research Office under Grant DAAL03-90-G-0108, and in part by the Advanced Research Projects Agency of the Department of Defense, monitored by the Air Force Office of Scientific Research under Contract F4999620-90-C-0014, and in part by the Naval Research Laboratory under Contact N00014-94-1G0003. This manuscript is submitted for publication with the understanding that the U.S. Government is authorized to reproduce and distribute reprints for Government purposes notwithstanding any copyright notation herein. The views and conclusions in this document are those of the authors and should not be interpreted as necessarily representing the official policies or endorsements, either expressed or implied, of the Army Research Office, the Advanced Research Projects Agency, or the U.S. Government.

L. Tong is with the Department of Electrical and Systems Engineering, University of Connecticut, U-157, Storrs, CT 06269-3157, USA.

$\mathrm{G}$. Xu is with the Department of Electrical and Computer Engineering, The University of Texas at Austin, Austin, TX 78712, USA.

B. Hassibi and T. Kailath are with the Information Systems Laboratory, Stanford University, Stanford, CA 94305, USA

IEEE Log Number 9407105.
II. Problem Formulation

\section{A. The Model}

We consider a discrete-time model for a baseband communication system with QAM signaling

$$
\begin{aligned}
& x(t)=\sum_{\alpha=-\infty}^{\infty} h(\alpha) u(t-\alpha)+n(t) \\
& u(t)=\sum_{k} s_{k} \delta(t-k T)
\end{aligned}
$$

where $x(\cdot)$ is the channel output process, $h(\cdot)$ is the channel impulse response, $\left\{s_{k}\right\}$ the sequence of information symbols, $1 / T$ the symbol transmission rate, and $n(\cdot)$ an additive noise process. The sampling rate is normalized to 1 .

The following conditions are assumed in the paper:

A1. The symbol interval $T$ is a known integer, $T>1$.

A2. The channel has a finite impulse response.

A3. $\left\{s_{k}\right\}$ is zero mean, and $E\left(s_{k} s_{l}^{*}\right)=\delta(k-l)$, where $\delta(t)$ is the discrete-time impulse function.

A4. $n(\cdot)$ is zero mean, white, and uncorrelated with $\left\{s_{k}\right\}$.

Remark: To study the channel identifiability conditions, we assume that the noise variance is known. However, such an assumption is not necessary for the blind channel identification algorithm proposed in this communication.

\section{B. Cyclostationarity Properties}

To obtain the relation between the output (cyclostationary) statistics and the channel transfer function, define the autocorrelation function of $x(\cdot)$ and its Fourier transform by

$$
\begin{aligned}
& r_{x}(t, \tau)=E\left(x(t) x^{*}(t-\tau)\right) \\
& S_{x}(t, \nu)=\sum_{\tau} r_{x}(t, \tau) e^{-j \nu \tau} .
\end{aligned}
$$

The autocorrelation functions (Fourier transforms of the autocorrelations) of $u(\cdot)$ and $n(\cdot)$ are denoted by $r_{u}(t, \tau)\left(S_{u}(t, \nu)\right)$ and $r_{n}(t, \tau)\left(S_{n}(t, \nu)\right)$, respectively. Under Assumptions A1-A4, it can be shown that

$$
\begin{aligned}
& S_{x}(t, \nu)=\left(\sum_{\alpha} h(\alpha) e^{-j \nu \alpha} S_{u}(t-\alpha, \nu)\right) H^{*}(\nu)+S_{n}(t, \nu)(3) \\
& S_{u}(t, \nu)=\sum_{k=-\infty}^{\infty} \delta(t-k T)
\end{aligned}
$$

where $H(\nu)$ is the discrete-time Fourier transform of $h(\cdot)$. Since $S_{u}(t, \nu)$ is a periodic function of $t$ with the period $T, S_{x}(t, \nu)$ is also periodic in $t$ with period $T$ when the noise process is widesense stationary, as assumed in this communication. Let $\Gamma_{x}^{(k)}(\nu)$ be the $k$ th Fourier coefficient of $S_{x}(t, \nu)$, referred to as the $k$ th $c y c l i c$ spectrum, given by

$$
\Gamma_{x}^{(k)}(\nu)=\sum_{t=0}^{T-1} S_{x}(t, \nu) e^{-j k(2 \pi / T) t}, \quad k=0, \cdots, T-1 .
$$

We have, from (3)

$$
\begin{aligned}
\Gamma_{x}^{(k)}(\nu)=H\left(\nu+k \frac{2 \pi}{T}\right) \Gamma_{u}^{(k)}(\nu) H^{*}(\nu)+\Gamma_{n}^{(k)}(\nu), & \\
& 0 \leq k \leq T-1
\end{aligned}
$$

where $\Gamma_{u}^{(k)}(\nu)$ and $\Gamma_{n}^{(k)}(\nu)$ are the $k$ th cyclic spectra of $u(\cdot)$ and $n(\cdot)$, respectively. Under Assumptions A3 and A4 and by (4), we 
can show that

$$
\Gamma_{u}^{(k)}(\nu)=1 \quad \Gamma_{n}^{(k)}(\nu)=T \sigma^{2} \delta(k)
$$

where $\sigma^{2}=E\left(n(t) n^{*}(t)\right)$. Hence, (6) becomes

$\Gamma_{x}^{(k)}(\nu)=H\left(\nu+k \frac{2 \pi}{T}\right) H^{*}(\nu)+T \sigma^{2} \delta(K)$,

$$
0 \leq k \leq T-1 \text {. }
$$

For notational convenience, let $\omega=\nu+k(2 \pi / T)$ and

$$
\Gamma^{(k)}(\omega)=\Gamma_{x}^{(k)}\left(\omega-k \frac{2 \pi}{T}\right)-T \sigma^{2} \delta(k)
$$

then we have

$$
\Gamma^{(k)}(\omega)=H(\omega) H^{*}\left(\omega-k \frac{2 \pi}{T}\right), \quad k=0, \cdots, T-1
$$

It is convenient to treat the problem of channel identifiability in the $z$-domain. Let

$$
H(z)=\sum_{k} h(k) z^{-k}
$$

With the correspondences $H(\omega) \leftrightarrow H(z), H^{*}(\omega-k \theta) \leftrightarrow$ $H^{*}\left(e^{-j k \theta}\left(1 / z^{*}\right)\right)$, and $\Gamma^{(k)}(\omega) \leftrightarrow \Gamma^{(k)}(z)$, we obtain the identification equations

$$
\Gamma^{(k)}(z)=H(z) H^{*}\left(e^{-j k(2 \pi / T)} \frac{1}{z^{*}}\right), \quad k=0, \cdots, T-1 .
$$

If $\gamma_{i \tau}^{(k)}$ is the coefficient of $z^{-\tau}$ in $\Gamma^{(k)}(z)$, we shall now relate it to the autocorrelation function $r_{x}(t, \tau)$, which can be estimated from the channel output in practice. Denoting the cyclic autocorrelation by

$$
\eta_{\tau}^{(k)}=\sum_{t=0}^{T-1} r_{x}(t, \tau) e^{-j k(2 \pi / T) t}
$$

the coefficient $\gamma_{\tau}^{(k)}$ is given by

$$
\gamma_{\tau}^{(k)}= \begin{cases}\eta_{\tau}^{(k)} e^{j k(2 \pi / T) \tau}, & \text { for } k \neq 0 \\ \eta_{\tau}^{(0)}-\sigma^{2} T \delta(\tau), & \text { for } k=0\end{cases}
$$

It is noted that, once $r_{x}(t, \tau)$ and $\sigma$ are obtained, $\gamma_{\tau}^{(k)}$ can be computed by applying the fast Fourier transform (FFT) to $r_{x}(t, \tau)$. The problem of channel identification is now equivalent to identifying $H(i)$ given $\Gamma^{(k)}(z)$.

III. NECESSARY AND SUfFICIENT CONDITIONS OF CHANNEL IDENTIFIABILITY

The issue of channel identifiability is related to the following question: given $\left\{\Gamma^{(k)}(z), k=0, \cdots, T-1\right\}$, to what extent can $H(z)$ be determined? It is clear from (12) that there is an inherent phase ambiguity for the identification in the sense that if $H(z)$ satisfies (12), so does $H(z) e^{j \phi}$ for any $\phi$. Such ambiguity has been noted in [1], and it is generally considered acceptable for communication applications. The more important question is whether this ambiguity is the only ambiguity. A linear time-invariant channel with a finite impulse response is therefore referred to as identifiable if it can be determined from (12) up to a multiplicative constant (phase).

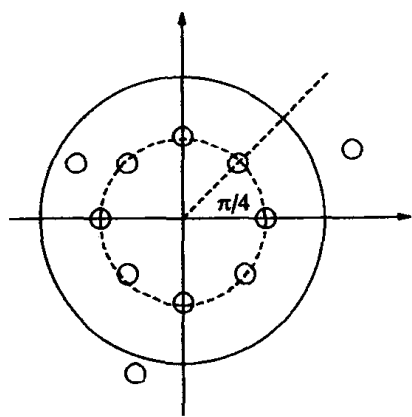

Fig. 1. A channel with uniformly $(\pi / 4)$-spaced zeros.

\section{A. A Condition on the Channel Transfer Function}

The identifiability condition on $H(z)$ relates to a simple notion of uniformly spaced zeros. A channel transfer function is said to have uniformly $\theta$-spaced zeros if a subset of zeros of $H(z)$ is uniformly spaced on a circle of the $z$-plane with angular spacing $\theta$, see Fig. 1 . Such a set of zeros must have the form $\left\{r e^{j \phi+\theta}, r e^{j \phi+2 \theta}, \cdots, r e^{j \phi+K \theta}=r e^{j \phi}\right\}$.

The following theorem on channel identifiability applies to a linear time-invariant FIR channel. A similar condition for stable minimal IIR channels can be found in [10].

Theorem 1: An FIR channel $H(z)$ is identifiable from $\left\{\Gamma^{(k)}(z)\right\}$ if and only if $H(z)$ does not have uniformly $(2 \pi / T)$-spaced zeros. Moreover, if the channel is identifiable, the zeros of $H(z)$ are the common zeros of the $\left\{\Gamma^{(k)}(z), k=0, \cdots, T-1\right\}$, i.e.,

$$
\mathcal{Z}(H(z))=\bigcap_{k} \mathcal{Z}\left(\Gamma^{(k)}(z)\right)
$$

where $\mathcal{Z}(H(z))$ stands for a set of zeros ${ }^{1}$ of $H_{(z)}$.

Before a proof is presented, we make a few comments along with two examples that provide some insights into the issue.

- The zeros of $H^{*}\left(e^{-j k \theta}\left(1 / z^{*}\right)\right)$ are reciprocals of the zeros of $H(z)$ rotated counterclockwise by $k \theta$, i.e., if $z_{0} \in \mathcal{Z}(H(z))$, then $\left(1 / z_{0}^{*}\right) e^{j k \theta} \in \mathcal{Z}\left(H^{*}\left(e^{-j k \theta}\left(1 / z^{*}\right)\right)\right)$.

- The zeros of $\Gamma^{(k)}(z)$ are related to the zeros of $H(z)$ by

$$
\mathcal{Z}\left(\Gamma^{(k)}(z)\right)=\mathcal{Z}(H(z)) \bigcup \mathcal{Z}\left(H^{*}\left(e^{-j k(2 \pi / T)} \frac{1}{z^{*}}\right)\right) .
$$

- The zero diagram of an identifiable channel is shown in Fig. 2(c), and the corresponding zeros of the $\Gamma^{(k)}(z)$ are shown in Fig. 2(a) and (b). The channel does not have uniformly spaced zeros and it is clear that the zeros of the channel can be obtained from the intersection of the zeros of the observation spectra

- An unidentifiable case is illustrated by Fig. 3 which shows two channels that have the same zero diagram for $\Gamma^{(k)}(z)$. Fig. 4 shows the corresponding impulse responses. In general, those zeros that are not $(2 \pi / T)$-spaced are uniquely determined. The $(2 \pi / T)$-spaced zeros are either minimum-phase or maximumphase zeros. If the channel has $m$ sets of $(2 \pi / T)$-spaced zeros, there are $2^{m}$ possible channels corresponding to the same output spectra. In a recent paper [13], Tugnait explored the implications of Theorem 1, and showed that a certain class of multipath channels is not identifiable using second-order statistics. The example given in Fig. 4 belongs to such channels. In this case, as suggested in [13], making use of higher order statistics may make the channel identifiable.

${ }^{1}$ Repeated zeros are considered as different elements of $\mathcal{Z}(H(z))$. 


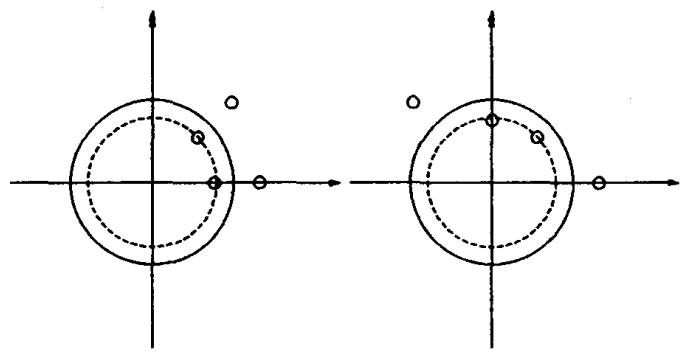

(a)

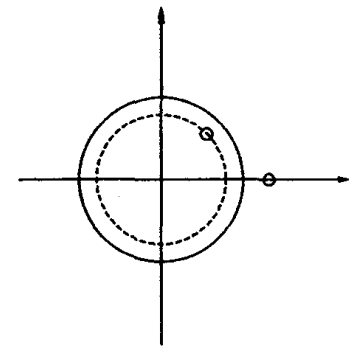

(c)

Fig. 2. (a) The zeros of $\Gamma^{(0)}(z)$. (b) The zeros of $\Gamma^{(1)}(z)$. (c) $\mathcal{Z}(H(z))=\mathcal{Z}\left(\Gamma^{(0)}(z)\right) \bigcap \mathcal{Z}\left(\Gamma^{(1)}(z)\right)$

Proof of Theorem 1: The necessity part is rather simple. The most obvious case is when all the zeros of $H(z)$ are uniformly $(2 \pi / T)$-spaced. In such a case

$$
\mathcal{Z}\left(\Gamma^{(k)}(z)\right)=\mathcal{Z}\left(\Gamma^{(0)}(z)\right), \quad \forall k
$$

It is then impossible to identify the zeros of $H(z)$ from $\mathcal{Z}\left(\Gamma^{(k)}(z)\right)$ since one cannot determine if a zero of $H(z)$ is inside or outside the unit circle. When a subset of $\mathcal{Z}(H(z))$ is uniformly $(2 \pi / T)$-spaced, this subset cannot be identified.

Suppose now that $H(z)$ does not have uniformly $(2 \pi / T)$-spaced zeros. We have, from (16)

$$
\bigcap_{k} \mathcal{Z}\left(\Gamma^{(k)}(z)\right)=\mathcal{Z}(H(z)) \bigcup \bigcap_{k} \mathcal{Z}\left(H^{*}\left(e^{-j k(2 \pi / T)} \frac{1}{z^{*}}\right)\right)
$$

We shall show that

$$
\bigcap_{k} \mathcal{Z}\left(H^{*}\left(e^{-j k(2 \pi / T)} \frac{1}{z^{*}}\right)\right)=\varnothing .
$$

Suppose that

$$
z_{0} \in \bigcap_{k} \mathcal{Z}\left(H^{*}\left(e^{-j k(2 \pi / T)} \frac{1}{z^{*}}\right)\right)
$$

Then

$$
z_{0} e^{-j k(2 \pi / T)} \in \mathcal{Z}\left(H^{*}\left(\frac{1}{z^{*}}\right)\right), \quad \forall k
$$

Therefore $\left\{z_{0}, z_{0} e^{-j(2 \pi / T)}, \cdots, z_{0} e^{-j(T-1)}(2 \pi / T)\right\}$ is a set of uniformly $(2 \pi / T)$-spaced zeros of $H^{*}\left(1 / z^{*}\right)$. Hence $H(z)$ has uniformly $(2 \pi / T)$-spaced zeros. This contradiction leads to (19) and the fact that the zeros of $H(z)$ can be identified from $\mathcal{Z}\left(\Gamma^{(k)}(z)\right)$ by (15).

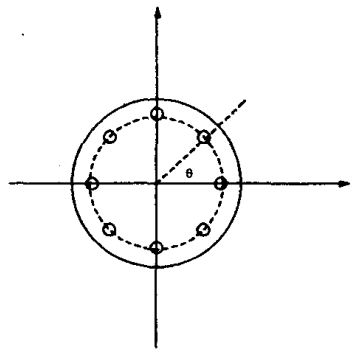

(a)

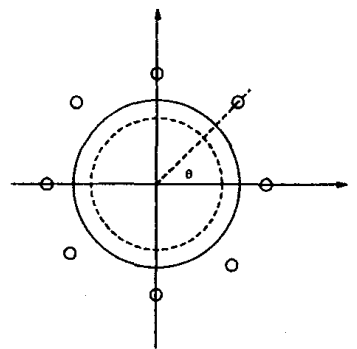

(b)

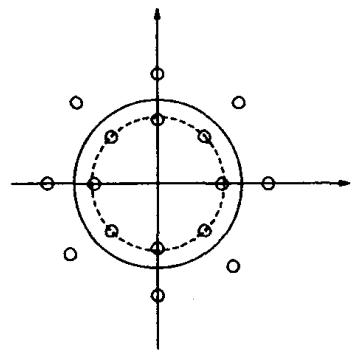

(c)

Fig. 3. (a) Two channels having uniformly $(\pi / 4)$-spaced zeros. (b) The zeros of $\Gamma^{(k)}(z)$ for all $k$.
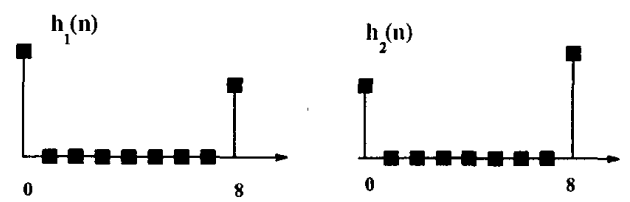

Fig. 4. Impulse responses of two unidentifiable channels.

\section{B. Conditions on the Output Spectra}

Theorem 1, unfortunately, is not very useful in practice in determining whether a given channel is identifiable, because $H(z)$ is itself unknown. The conditions presented next, though a direct consequence of Theorem 1, can be verified based on the output spectra.

Lemma 1: An $L$ th-order FIR channel $H(z)$ is identifiable from $\left\{\Gamma^{(k)}(z)\right\}$ if and only if $\left\{\Gamma^{(k)}(z)\right\}$ have exactly $L$ common zeros.

Proof: The necessary part is immediate from (15). For the sufficient part, we observe from (12) that $\left\{\Gamma^{(k)}(z)\right\}_{k=0}^{T-1}$ must have at least $L$ common zeros. Suppose that the channel is not identifiable, i.e., $H(z)$ has a set of uniformly $(2 \pi / T)$-spaced zeros. Then, $\left\{\Gamma^{(k)}(z)\right\}$ have the same two sets of uniformly $(2 \pi / T)$-spaced zeros for all $k$, where one set comes from $H(z)$, and the other comes from $H^{*}\left(e^{-j 2 k \pi / T} / z^{*}\right)$. Therefore, $\left\{\Gamma^{(k)}(z)\right\}_{k=0}^{T-1}$ must have more than $L$ common zeros.

The condition for the existence of common zeros can be verified conveniently by checking the ranks of the generalized Sylvester resultants proposed by Kung, Kailath, and Morf [8], [2].

Lemma 2 ([8]): Given an $r \times r$ matrix polynomial

$$
C(z)=\sum_{i=0}^{m} C_{i} z^{i}
$$

and a $q \times r$ matrix polynomial

$$
\boldsymbol{D}(z)=\sum_{i=0}^{m} \boldsymbol{D}_{i} z^{i}
$$


we have

$$
\operatorname{rank}\left(S_{k}(D, C)\right)=(r+q) k-\sum_{i: v_{i}<k}\left(k-\nu_{i}\right)
$$

where $S_{k}(D, C)$ is the generalized Sylvester resultant matrix of $C(z)$ and $\boldsymbol{D}(z)$ of order $k$

$$
S_{k}(\boldsymbol{D}, \boldsymbol{C})=\underbrace{\left(\begin{array}{ccccccc}
\boldsymbol{D}_{0} & \boldsymbol{D}_{1} & \cdots & \boldsymbol{D}_{m} & \mathbf{0} & \cdots & \mathbf{0} \\
\boldsymbol{C}_{0} & \boldsymbol{C}_{1} & \cdots & \boldsymbol{C}_{m} & \mathbf{0} & \cdots & \mathbf{0} \\
\mathbf{0} & \boldsymbol{D}_{0} & \boldsymbol{D}_{1} & \cdots & \boldsymbol{D}_{L} & \cdots & \mathbf{0} \\
\mathbf{0} & \boldsymbol{C}_{0} & \boldsymbol{C}_{1} & \cdots & \boldsymbol{C}_{L} & \cdots & \mathbf{0} \\
\vdots & \vdots & \ddots & \ddots & \ddots & \ddots & \vdots \\
\mathbf{0} & \cdots & \mathbf{0} & \boldsymbol{D}_{0} & \boldsymbol{D}_{1} & \cdots & \boldsymbol{D}_{m} \\
\mathbf{0} & \cdots & \mathbf{0} & \boldsymbol{C}_{0} & \boldsymbol{C}_{1} & \cdots & \boldsymbol{C}_{m}
\end{array}\right)}_{(m+k) \text { blocks }}
$$

and $\nu_{i}, i=1, \cdots, r$ are the so-called dual dynamic indices (see [3]) of $\boldsymbol{T}(z)=\boldsymbol{D}(z) C^{-1}(z)$.

Proof: See [2].

With Lemmas 2 and 1, we obtain the following necessary and sufficient condition on the observation spectra.

Theorem 2: An $L$ th-order FIR channel $H(z)$ is identifiable from the $\left\{\Gamma^{(k)}(z)\right\}$ if and only if

$$
\operatorname{rank}\left(S_{k}(\boldsymbol{D}, c)\right)=k+L, \quad \text { for all } k \geq L
$$

where $\boldsymbol{S}_{k}(D, c)$ is the generalized Sylvester matrix constructed from

$$
\begin{aligned}
\boldsymbol{D}(z) & =\left[\Gamma^{(1)}(z), \cdots, \Gamma^{(T-1)}(z)\right]^{t} \\
c(z) & =\Gamma^{(0)}(z) .
\end{aligned}
$$

Proof: We first note that, in applying Lemma 2 to $(\boldsymbol{D}(z), c(z))$, we deal with a $(T-1) \times 1$ polynomial vector

$$
\boldsymbol{T}(z)=\frac{1}{c(z)} D(z) \text {. }
$$

Next, we argue that there are exactly $L$ common zeros among $\left\{\Gamma^{(k)}(z)\right\}$ if and only if the minimal state-space realization of $\boldsymbol{D}(z) / c(z)$ has order $L$ (see [7]), i.e., $\sum_{i} \nu_{i}=L$. By applying Lemma 2 with $r=1$, we conclude that the channel is identifiable if and only if

$$
\left.\operatorname{rank}\left(\boldsymbol{S}_{k}(\boldsymbol{D}, c)\right)=1+T-1\right) k-\sum_{i=1}^{T-1}\left(k-\nu_{i}\right)
$$

which leads directly to (23)

Remark: An interesting implication of this theorem and Lemma 2 is that, for $T=2$, the channel order can be determined by the changes in rank of the corresponding Sylvester resultants. A channel is of order $L$ if the rank of the Sylvester resultant $\boldsymbol{S}_{k}(D, c)$ increases by 2 for $k \leq L$ and by 1 for $k>L$. Note that both $D$ and $c$ can be obtained from the observation.

\section{A Necessary and Sufficient Condition in the Time Domain}

The necessary and sufficient conditions in the frequency domain turn out to be very useful in deriving a necessary and sufficient condition in the time domain. We shall now show that the sufficient condition given earlier in [12] is in fact also necessary. This result establishes a relation between the time-domain and frequency-domain approaches. A similar result was shown independently in [9] by a different method.

The sufficient condition given in [12] is based on a vector representation of the cyclostationary process. This vector representation is in fact a special case of the so-called translation series representation (TSR) of cyclostationary processes derived by Gardner and Franks
[5], which represents a cyclostationary process by a set of jointly stationary processes.

If an $(L+1) T \times 1$ vector $\boldsymbol{x}(k)$ is formed by

$$
\begin{array}{r}
\boldsymbol{x}(k)=[x(k T), \cdots, x(k T+T-1) \mid \\
\cdots \mid x((k-L) T), \\
\cdots, x((k-L+1) T-1)]^{l}
\end{array}
$$

then the vector $\boldsymbol{x}(k)$ is related to a vector of input symbols $\left\{s_{k}\right\}$ by a memoryless time-invariant matrix filter $\boldsymbol{H}$ of dimension $(L+$ 1) $T \times(2 L+1)$ (plus noise)

$$
\boldsymbol{x}(k)=\boldsymbol{H} \boldsymbol{s}(k)+\boldsymbol{n}(k)
$$

where

$$
\begin{aligned}
\boldsymbol{s}(k)= & {\left[s_{k}, s_{k-1}, \cdots, s_{k-2 L-1}\right]^{t} } \\
\boldsymbol{n}(k)= & {[n(k T), \cdots, n(k T+T-1)|\cdots| n((k-L) T),} \\
& \cdot \cdots, n((k-L+1) T-1)]^{t}
\end{aligned}
$$

and the channel parameter matrix turns out to be a Sylvester resultant matrix

$$
\boldsymbol{H}=\underbrace{\left(\begin{array}{ccccccc}
\boldsymbol{h}_{0} & \boldsymbol{h}_{1} & \cdots & \boldsymbol{h}_{L} & \mathbf{0} & \cdots & \mathbf{0} \\
\mathbf{0} & \boldsymbol{h}_{0} & \boldsymbol{h}_{1} & \cdots & \boldsymbol{h}_{L} & \cdots & \mathbf{0} \\
\vdots & \vdots & \ddots & \ddots & \ddots & \ddots & \vdots \\
\mathbf{0} & \cdots & \mathbf{0} & \boldsymbol{h}_{0} & \boldsymbol{h}_{1} & \cdots & \mathbf{h}_{L}
\end{array}\right)}_{2 L+1}
$$

where $\boldsymbol{h}_{k}=[h(k T), h(k T+1), \cdots, h(k T+T-1)]^{l}$.

It was shown in [12] that the channel is identifiable if $\boldsymbol{H}$ has full column rank. We shall now show that this condition is also necessary. The next theorem incorporates the explicit identification formula given in [12].

Theorem 3: The channel impulse response is identifiable if and only if matrix $\boldsymbol{B}$ has full column rank. If the channel is identifiable, the channel parameter matrix $\boldsymbol{H}$ is given by

$$
\boldsymbol{H}=\alpha \boldsymbol{U} \boldsymbol{\Lambda} \boldsymbol{V}^{H}
$$

where $\alpha$ is an unknown constant, $\boldsymbol{U}$ and $\boldsymbol{\Lambda}$ are obtained from the singular value decomposition (SVD) of

$$
\begin{gathered}
\boldsymbol{R}_{0}=E\left(\boldsymbol{x}(t) \boldsymbol{x}^{H}(t)\right)-E\left(\boldsymbol{n}(t) \boldsymbol{n}^{T}(t)\right) \\
\boldsymbol{R}_{0}=\boldsymbol{U} \boldsymbol{\Lambda}^{2} \boldsymbol{U}^{H}
\end{gathered}
$$

and $\boldsymbol{V}$ is obtained from the left singular vector $\boldsymbol{p}$ associated with the smallest singular value of

$$
\begin{gathered}
\boldsymbol{R}_{1}=\boldsymbol{T}\left(E\left(\boldsymbol{x}(t) \boldsymbol{x}^{H}(t-1)\right)-E\left(\boldsymbol{n}(t) \boldsymbol{n}^{H}(t-1)\right)\right) \boldsymbol{T}^{H} \\
\boldsymbol{V}=\left[\boldsymbol{p}, \boldsymbol{R}_{1} \boldsymbol{p}, \cdots, \boldsymbol{R}_{1}^{d-1} \boldsymbol{p}\right]
\end{gathered}
$$

where $\boldsymbol{T}$ is (the so-called Mahalanobis transform)

$$
\boldsymbol{T}=\boldsymbol{\Lambda}^{-1} U^{H} \text {. }
$$

Proof: The proofs of the sufficiency and of the identification formula are given in [12]. To show the necessary part, we note that $\boldsymbol{H}$ is a Sylvester resultant of order $L+1$. Let $a_{i}(z)$ be the polynomial constructed from the $(i+1)$ th row of $\boldsymbol{H}$

$a_{i}(z)=h(i)+h(i+T) z^{-1}+\cdots+h(i+L T) z^{-L}$,

$$
i=0, \cdots, T-1 \text {. }
$$

Using Lemma 2, again with $r=1$, we have

$$
\operatorname{rank}(\boldsymbol{H})=L+1+\sum_{i} \nu_{i}
$$


where $\nu_{i}$ are the dual dynamic indices of

$$
\frac{1}{a_{T-1}(z)}\left[a_{0}(z), \cdots, a_{T-2}(z)\right]^{t} \text {. }
$$

If $\boldsymbol{H}$ does not have full column rank, i.e., $\operatorname{rank}(\boldsymbol{H})<2 L+1$, then $\sum \nu_{i}<L$. This implies that the $\left\{a_{i}(z)\right\}$ share common zeros, i.e., there exists a $z_{0}=r_{0} e^{j \phi_{0}}$ such that

$$
a_{i}\left(z_{0}\right)=0, \quad i=0, \cdots, T-1 .
$$

Note that

$$
H(z)=\sum_{i=0}^{T-1} a_{i}\left(z^{T}\right) z^{-i}
$$

Let

$$
p_{k}=r_{0}^{1 / T} e^{j\left[\left(\phi_{0}+2 k \pi\right) / T\right]}, \quad k=0, \cdots, T-1 .
$$

Clearly, $p_{k}^{T}=z_{0}$ and

$$
H\left(p_{k}\right)=\sum_{i=0}^{T-1} a_{i}\left(p_{k}^{T}\right) p_{k}^{-i}=\sum_{i=0}^{T-1} a_{i}\left(z_{0}\right) p_{k}^{-i}=0 .
$$

Therefore, $\left\{p_{k}, k=0, \cdots, T-1\right\}$ are zeros of $H(z)$ or $H(z)$ has uniformly $(2 \pi / T)$-spaced zeros. By Theorem 1 , the channel is not identifiable.

Theorem 3 also demonstrates that the time-domain algorithm proposed in [12] is able to identify all identifiable channels.

\section{A FREQUENCY-DOMAIN ALGORITHM}

We now consider the problem of identifying the channel using two output cyclic spectra $\Gamma^{\left(k_{1}\right)}(z)$ and $\Gamma^{\left(k_{2}\right)}(z)$. Suppose that $\Gamma^{\left(k_{1}\right)}(z)$ and $\Gamma^{\left(k_{2}\right)}(z)$ have exactly $L$ common zeroes, i.e., $H(z)$ is identifiable from $\Gamma^{\left(k_{1}\right)}(z)$ and $\Gamma^{\left(k_{2}\right)}(z)$, it is easily shown from (10) that, for any

$$
\begin{gathered}
Q(z)=\sum_{n=0}^{L} q_{n} z^{-n} \\
\Gamma^{\left(k_{1}\right)}(z) Q^{*}\left(e^{j k_{2}(2 \pi / T)} \frac{1}{z^{*}}\right)-\Gamma^{\left(k_{2}\right)} Q^{*}\left(e^{j k_{1}(2 \pi / T)} \frac{1}{z^{*}}\right)=0
\end{gathered}
$$

if and only if $Q(z)=\alpha H(z)$ for some constant $\alpha$. By equating coefficients of powers of $z$ in (41) with $Q(z)=H(z)$, we get

$$
\boldsymbol{G}(k 1, k 2)[h(L), \cdots, h(0)]^{H}=0
$$

where the $(3 L+1) \times(L+1)$ matrix $G(k 1, k 2)$ is defined by (see (43) at the bottom of page).

Moreover, the dimension of the null space of $G(k 1, k 2)$ satisfies

$$
\operatorname{dim} \mathcal{N}(G(k 1, k 2))=1 .
$$

With a slight generalization, we have the following necessary and sufficient condition that involves all the $\Gamma^{(k)}(z)$.
Theorem 4: An $L$ th-order FIR channel $H(z)$ is identifiable from the $\left\{\Gamma^{(k)}(z)\right\}$ if and only if

$$
\operatorname{dim}(\mathcal{N}(G))=1 .
$$

where

$$
\begin{aligned}
G= & {\left[G^{t}(0,1), \cdots, G^{t}(0, T-1), G^{t}(1,2),\right.} \\
& \left.\times \quad \cdots, G^{t}(1, T-1), \cdots, G^{t}(T-2, T-1)\right]^{t} .
\end{aligned}
$$

Moreover

$$
\boldsymbol{G}[h(L), \cdots, h(0)]^{H}=\mathbf{0} .
$$

Remark: Equations (45) and (47) form the foundation of the frequency-domain blind channel identification algorithm first proposed in [6].

Although the channel can be identified from the zeros of the cyclic spectra of the channel output suggested by Theorem 1, such identification is sensitive to noise and estimation error. We shall now derive a channel identification algorithm based on the frequencydomain formulation, and it is motivated by minimizing the effect of noise and estimation error in some sense.

From the observations, we can obtain the estimated cyclic autocorrelation functions $\hat{\eta}_{\tau}^{\left(k_{1}\right)}, \hat{\eta}_{\tau}^{\left(k_{2}\right)}$. The estimated output spectra $\hat{\Gamma}^{\left(k_{1}\right)}(z)$ and $\hat{\Gamma}^{\left(k_{2}\right)}(z)$ are given by

$$
\hat{\Gamma}^{\left(k_{j}\right)}(z)=\sum_{i=-L}^{L} \hat{\gamma}_{i}^{\left(k_{j}\right)} z^{-i}, \quad j=1,2
$$

where, from (14)

$$
\hat{\gamma}_{\tau}^{\left(k_{j}\right)}=\hat{\eta}_{\tau} e^{j k_{i}(2 \pi / T) \tau}, \quad i=1,2 .
$$

The estimate $\hat{H}(z)$ of $H(z)$ is obtained via the following optimization problem:

$$
\begin{aligned}
\hat{H}(z)=\underset{\mathbf{H}(\mathrm{z})}{\operatorname{argmin}} \| \hat{\Gamma}^{\left(\mathrm{k}_{1}\right)}(\mathrm{z}) & \mathrm{H}^{*}\left(\mathrm{e}^{\mathrm{j \textrm {k } _ { 2 } ( 2 \pi / \mathrm { T } )}} \frac{1}{\mathrm{z}^{*}}\right) \\
& -\hat{\Gamma}^{\left(k_{2}\right)}(z) H^{*}\left(e^{j k_{1}(2 \pi / T)} \frac{1}{z^{*}}\right) \|^{2} .
\end{aligned}
$$

The solution

$$
\hat{H}(z)=\sum_{n=0}^{L} \hat{h}(n) z^{-n}
$$

to this minimization problem is given by

$$
[\hat{h}(L), \cdots, \hat{h}(0)]^{H}=\underset{\mathbf{u} \neq \mathbf{0}}{\operatorname{argmin}}\left\|\hat{\boldsymbol{G}}\left(\mathrm{k}_{\mathbf{1}}, \mathrm{k}_{2}\right) \boldsymbol{u}\right\|_{2}^{2}
$$

where $\hat{\boldsymbol{G}}\left(k_{1}, k_{2}\right)$ is the estimated $\boldsymbol{G}\left(k_{1}, k_{2}\right)$ defined in (43). The optimal solution (in the sense of (51)) is given by

$$
[\hat{h}(L), \cdots, \hat{h}(0)]^{H}=v_{L+1}
$$

$\boldsymbol{G}(k 1, k 2)=\left(\begin{array}{cccc}\gamma_{-L}^{\left(k_{1}\right)} e^{j L k_{2}(2 \pi / T)}-\gamma_{-L}^{\left(k_{2}\right)} e^{j L k_{1}(2 \pi / T)} & & \\ \gamma_{-L+1}^{\left(k_{1}\right)} e^{j L k_{2}(2 \pi / T)}-\gamma_{-L+1}^{\left(k_{2}\right)} e^{j L k_{1}(2 \pi / T)} & \gamma_{-L}^{\left(k_{1}\right)} e_{-L}^{j(L-1) k_{2}(2 \pi / T)-\gamma\left(k_{2}\right)} e^{j(L-1) k_{1}(2 \pi / T)} & & \\ \vdots & \gamma_{-L+1}^{\left(k_{1}\right)} e^{j(L-1) k_{2}(2 \pi / T)}-\gamma_{-L+1}^{\left(k_{2}\right)} e^{j(L-1) k_{1}(2 \pi / T)} & \ddots & \\ \vdots & \vdots & \ddots & \gamma_{-L}^{\left(k_{1}\right)}-\gamma_{-L}^{\left(k_{2}\right)} \\ \gamma_{L}^{\left(k_{1}\right)} e^{j L k_{2}(2 \pi / T)}-\gamma_{L}^{\left(k_{2}\right)} e^{j L k_{1}(2 \pi / T)} & \vdots & \vdots & \gamma_{-L+1}^{\left(k_{1}\right)}-\gamma_{-L+1}^{\left(k_{2}\right)} \\ & \gamma_{L}^{\left(k_{1}\right)} e^{j L k_{2}(2 \pi / T)}-\gamma_{L}^{\left(k_{2}\right)} e^{j L k_{1}(2 \pi / T)} & \ddots & \vdots \\ & & \ddots & \vdots \\ & & & \gamma_{L}^{\left(k_{1}\right)}-\gamma_{L}^{\left(k_{2}\right)}\end{array}\right)$. 
where $\boldsymbol{v}_{L+1}$ is the right singular vector of $\hat{\boldsymbol{G}}\left(k_{1}, k_{2}\right)$ associated with the $(L+1)$ th singular value.

The choice of $k_{1}$ and $k_{2}$ may affect the performance of the algorithm. However, it is not difficult to combine the cyclic statistics for some or all (distinct) $k_{1}$ 's and $k_{2}$ 's. In doing so, the criterion in (51) can be modified by replacing $\hat{G}\left(k_{1}, k_{2}\right)$ by

$$
\begin{aligned}
\hat{G}=\left[G^{t}(0,1),\right. & \cdots, \hat{G}^{t}(0, T-1), \hat{G}^{l}(1,2), \\
& \left.\cdots, \hat{G}^{l}(1, T-1), \cdots, \hat{G}^{t}(T-2, T-1)\right]^{t} .
\end{aligned}
$$

Such modification may improve the performance.

\section{CONCluding Remarks}

We established several different necessary and sufficient conditions for the identifiability of a possibly nonminimum phase channel from its output cyclic autocorrelation functions. In comparison to the timedomain approach presented earlier in [12], the frequency-domain approach to the channel identification problem gives more insight into the issue of channel identifiability. It also provides the basis for new channel identification algorithms.

\section{ACKNOWLEDGMENT}

The authors wish to thank $\mathrm{H}$. Liu at the University of Texas at Austin for his comments.

\section{REFERENCES}

[1] M. Goursat, A. Benveniste, and G. Ruget, "Robust identification of a nonminimum phase system: Blind adjustment of a linear equalizer in data communication," IEEE Trans. Automat. Contr., vol. AC-25, no. 6 pp. 385-399, June 1980 .

12] R. R. Bitmead, S. Y. Kung, B. D. O. Anderson, and T. Kailath, "Greatest common divisors via generalized Sylvester and Bezout matrices," IEEE Trans. Automat. Contr., vol. AC-23, no. 6, pp. 1043-1047, Dec. 1978.

[3] G. D. Forney, "Minimal bases of rational vector spaces, with applications to multivariable linear systems," SIAM J. Contr., vol. 13 , no. 3 , pp. 493-520, May 1975.

[4] W. Gardner, "A new method of channel identification," IEEE Trans. Commun., vol. 39, no. 6, pp. 813-817, June 1991.

[5] W. A. Gardner and L. E. Franks, "Characterization of cyclostationary random signal processes," IEEE Trans. Inform. Theory, vol. IT-21, no. I, pp. 4-14, Jan. 1975.

[6] B. Hassibi, personal communication, Aug. 1992.

[7] T. Kailath, Linear Systems. Englewood Cliffs, NJ: Prentice-Hall, 1980

[8] S. Y. Kung, T. Kailath, and M. Morf, "A generalized resultant matrix for polynomial matrices," in Proc. IEEE Conf. on Decision and Control, 1976, pp. 892-895.

[9] Y. Li and Z. Ding, "Blind channel identification based on secondorder cyclostationary statistics," in Proc. IEEE Int. Conf. on Acoustics, Speech, and Signal Processing, vol. IV (Minneapolis, MN, Apr. 1993), pp. $81-84$.

[10] L. Tong, "Blind channel identification using cyclic spectra," in Proc. 26th Conf. on Information Sciences and Systems (Princeton, NJ, Mar. 1992), pp. 711-716.

[11] L. Tong, G. Xu, and T. Kailath, "A new approach to blind identification and equalization of multipath channels," in 25th Asilomar Conf. (Pacific Grove, CA, Nov. 1991).

[12] — "Blind identification and equalization based on second-order statistics: A time domain approach," IEEE Trans. Inform. Theory, vol. 40, no. 2, Mar. 1994.

[13] J. K. Tugnait, "On blind identifiability of multipath channels using fractional sampling and second-order cyclostationary statistics," in Proc. 1993 Global Commun. (Houston, TX, Dec. 1993).

\section{Recursive Filtering and Smoothing for Reciprocal Gaussian Processes-Pinned Boundary Case}

E. Baccarelli, R. Cusani, Member, IEEE, and G. Di Blasio, Associate Member, IEEE

Abstract- The least square estimation problem for pinned-to-zero discrete-index reciprocal Gaussian processes in additive white noise is solved, thus completing and extending some previous results available in the literature. In particular, following the innovations approach a (finite) set of recursive equations is obtained for the filter and for the three standard classes of smoothers (fixed-point, fixed-interval, fixed-lag). Recursive expressions for the mean square performance of the proposed estimators are also given.

Index Terms-Reciprocal processes, innovations method, recursive estimators.

\section{INTRODUCTION}

A discrete-index multivariate real reciprocal Gaussian random process (RGP) $\left\{x(k) \in R^{n}, M \leq k \leq N\right\}$ defined on an assigned probability space $(\Omega, \mathrm{A}, \mathrm{P})$ is described by the well-known selfadjoint second-order noncausal difference model [1]

$$
\begin{array}{r}
M_{0}(k) x(k)-M_{+}(k) x(k+1)-M_{+}^{T}(k-1) x(k-1)=e(k), \\
M+1 \leq k \leq N-1
\end{array}
$$

where $\left\{M_{0}(k)\right\},\left\{M_{+}(k)\right\}$ are deterministic sequences of $n \times n$ matrices. The conjugate process

$$
\left\{e(k) \in R^{n},(M+1) \leq k \leq(N-1)\right\}
$$

is bi-orthogonal to $\{x(k)\}$, that is,

$$
E\left\{x(k) e^{T}(s)\right\}=I \delta(k, s),
$$

and its covariance matrix is given by [1, eqs. (3.4a), (3.4b)]. Boundary random values with singular probability measures (pinned-to-zero) are considered in this paper, i.e., $x(M)=x(N)=0, P$-a.s.

It is assumed that the sequence $\{x(k)\}$ is amplitude-modulated and then sequentially transmitted through a noisy communication channel for increasing values of the index $k$. As a consequence, the observed sequence $\left\{y(k) \in R^{r}\right\}$ is modeled as

$$
y(k)=\Gamma(k) x(k)+w(k), \quad M+1 \leq k \leq N-1
$$

where $\left\{w(k) \in R^{r}\right\}$ is an additive white Gaussian noise (AWGN) process, independent of $\{x(k)\}$, with covariance matrices $\{R(k)\} ;\{\Gamma(k)\}$ are known and uniformly limited $r \times n$ matrices. It is also assumed that the matrices $\left\{M_{0}(k)\right\}$ and $\{R(k)\}$ are nonsingular.

In this correspondence, the problem of estimating a pinned-to-zero RGP in AWGN is addressed. Pinned-to-zero boundary conditions represent an important subclass of the more general Dirichlet boundary conditions [1]. In this case, an RGP does not always admit a wellbehaved first-order causal white innovations representation over the whole parameter space (e.g., including both ending points) giving a Markov version (in the stochastic sense) of the assigned process. As a consequence, the pertaining estimation problems cannot be directly

Manuscript received June 29, 1993; revised July 4, 1994.The material in this paper was presented at the IEEE International Symposium on Information Theory, Trondheim, Norway, June 27-July 1, 1994

The authors are with the INFOCOM Department, University of Rome 'La Sapienza,' 00184 Rome, Italy.

IEEE Log Number 9407260 